\title{
BEAM OPTICS STUDIES FOR THE CEBAF ACCELERATOR'
}

\author{
$\underline{\text { S.A. Bogacz }}$ and V.A. Lebedev, Thomas Jefferson National Accelerator Facility, Newport News, VA
}

\begin{abstract}
Measurements of differential orbits excited by two pairs of horizontal and vertical correctors, and by a change of the energy gain for the first one of two superconducting linacs allowed us to perform on-line control of the machine optics, which provided valuable information for studying optics discrepancies. Off-line analysis of the data has uncovered a number of malfunctioning hardware pieces, e.g. improperly functioning BPMs and incorrectly focusing quadrupoles. It also indicated that our dipoles have significant focusing terms, which have to be taken into account to build a predictable optics. The analysis resulted in a significantly improved optics model for the CEBAF recirculator. The new optics preserved desired lattice architecture and orthogonal tunability. The presented approach was proven very successful in minimizing required tuning time and in building a more accurate theoretical model of beam transport for the CEBAF accelerator.
\end{abstract}

\section{INTRODUCTION}

The CEBAF accelerator [1] is a five-pass $\mathrm{CW}$ recirculator with beam power up to $800 \mathrm{~kW}$. It consists of a $56 \mathrm{MeV}$ injector, two superconducting linacs of $500 \mathrm{MeV}$ energy gain, and nine arcs, which connect the linacs for beam recirculation for total beam energy of $5.056 \mathrm{GeV}$. Logically, the machine is separated into the following regions [2]: injector, North and South linacs, nine $180^{\circ}$ bend recirculation arcs with associated entrance and exit matching regions, and the spreader and recombiner regions at the ends of each linac, which separate particles of different energies or merge them for reinjection into the succeeding linac. After acceleration to the desired energy, the beam can be split and directed to three experimental halls for nuclear physics experiments. The path traversed to full energy is more than $6 \mathrm{~km}$ in length, over which the beam is focused by about 700 quadrupoles. Each quadrupole is independently powered, which on one hand creates many possibilities for machine optics, but on the other hand complicates machine tuning and operation processes.

The large scale of the accelerator requires high field accuracy of the magnets. The design reproducibility specifications for the quadrupoles $\left(\sim 2 \times 10^{-4}\right)$ have not been met in the real machine, which caused significant optics changes during the machine commissioning [3]. The main

\footnotetext{
* This work was supported by U.S. D.O.E. contract \#DE-AC0584ER40150.

- bogacz@jlab.org
}

motivation for optics redesign was to reduce error sensitivity of the machine optics and to create a set of orthogonal knobs for machine tuning

We identified three major sources of machine irreproducibility. The first one is adjustments of the accelerating profile, which is always non-uniform and is frequently changed when one or a few cavities cannot support the required accelerating gradient. In this case the focusing for the first pass is corrected, while the higher passes are subject to a significant betatron mismatch. The second source is a path-length adjustment. To keep higher passes on the 'crest' of the accelerating wave the path-length has to be stabilized at the level of $0.25 \mathrm{~mm}\left(\sim 0.5^{0}\right)$. That calls for frequent path-length adjustments to account for seasonal and weather variations. These adjustments cause the vertical beta function mismatch due to significant changes of the vertical focusing in the correction chicane. The third source is an overall deficiency in machine reproducibility, due to substantial temporal drifts of the quadrupole gradients, over a period of a few days or occasionally of a few hours. The first two sources were addressed at the commissioning time, which brought the machine to a reasonable state enabling initial beam delivery for physics. Although we could deliver acceptable quality beam to the experiments at that time, the machine optics was not understood sufficiently, which significantly hampered machine operation and beam optics software tools.

To build an adequate model of the machine optics and to gain some insight into sources of machine irreproducibility, systematic optics studies were initiated. Most of their findings are reported in this article.

\section{DIFFERENTIAL ORBIT ANALYSIS}

Two efforts to correct accelerator optics were undertaken. The first one is an envelope match in the injector. It is performed by measuring the vertical and horizontal beam sizes with beam profile monitors at four locations in the injector. Then adjustments of four upstream quads are made to match beam sizes to the injector Twiss functions. The second step involves tracking of machine optics with the differential orbit measurements, which serves as our main instrument for optics studies. The beam position measurement system includes approximately 800 BPMs. This large number of BPMs allows us to perform optics measurements, which are much more detailed than those done by any other available method.

To get complete measurements of linear optics one needs to use at least five sources of the beam orbit excitation: two horizontal correctors, two vertical correctors and an energy corrector. The best resolution for betatron motion is achieved when the effects of kicks excited by each 
corrector pair are orthogonal. That implies that correctors have to be shifted in betatron phase by $(1+2 n) \pi / 4$ [3]. Fast data acquisition for differential measurements was the key to the success of the project. Special data acquisition software was written to make measurements as fast as possible. The measurements are fully automated and it now takes about three minutes to perform measurements for the entire machine. This has allowed us to get the measurements routinely, every day at system checks or by a request from the Beam Transport Team.

To optimize the amplitude of excited betatron perturbation with respect to adiabatic damping, the exciting correctors were chosen at the beginning of Arc 1 (in the 556 $\mathrm{MeV}$ region). The energy perturbation was created by changing the accelerating gradient of eight RF cavities at the end of the first superconducting linac. The strengths of the above excitations were adjusted so that the resulting beam motion had initial transverse amplitude of about 3 $\mathrm{mm}$, which after acceleration (by a factor of ten) died down to about $1 \mathrm{~mm}$ due to the adiabatic damping.

A complete differential measurement, as illustrated in Figure 1, results in six independent differential orbits: two horizontal and two vertical differential orbits excited by correctors, and the horizontal and vertical dispersive orbits excited by the energy change. To reach a better clarity Figure 1 shows only the measurement restricted to Arc 2 and the downstream second pass in the North linac. Markers indicate actual measurement data, while continuous lines represent differential orbits predicted by the updated model of the machine optics.

Early post-commissioning experience [3] revealed serious discrepancies between predictions of the optics model and actual beam displacements in the machine. To 'pin down' possible sources of discrepancies, careful analysis of differential orbits through the entire machine was performed. We found out that the major sources of optics irreproducibility were linked to focusing effects of bending dipoles and several quads exhibiting few-percent focusing errors. The effect was particularly large for vertical dipoles of spreaders and recombiners, which were not measured with sufficient accuracy before installation. Simultaneous fitting of six independent difference orbits by varying the focusing terms of each dipole in the spreaders and recombiners allowed us to find a unique set of body gradients for all dipole magnets. A similar process was performed for the horizontal dipoles of nine arcs, but in

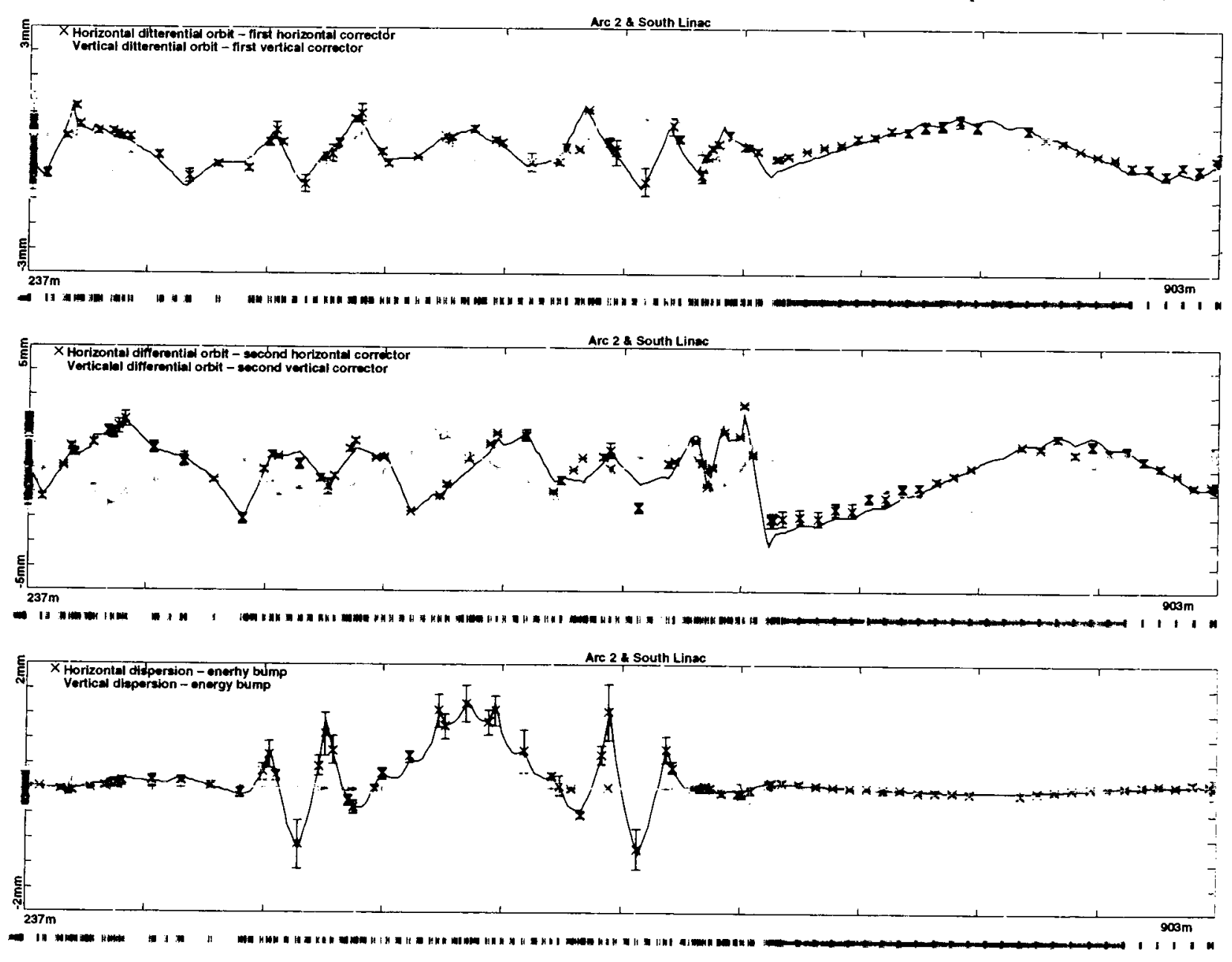

Figure 1: Example of differential orbit analysis for Arc 2 - complete set of six orbits - optics model vs measurement 
this case we were able to achieve a reasonable fit assuming that all dipoles of a single arc have identical focusing properties.

Extracted this way body gradients were used to update the optics mode. Improved status of the machine optics did not require betatron correction in the spreaders and the 'orthogonal knobs' for betatron match were left in the recombiners only. Another benefit of the improved optics model was reliable operation of an automated steering program, AutoSteer [5], which would not function properly without updating our initial 'crude' optics.

Careful testing and recalibration of power supplies for quads were performed in parallel with optics studies. After this process was complete the focusing of practically all quads corresponded to the design values. Currently, we are left with only four quadrupoles for which values are fudged by about $10 \%$ to fix the machine optics.

Another useful spin-off of optics studies was a test of the BPMs. The study indicated four BPMs, which exhibited incorrect (up to 50\%) differential displacement. Further improvements of the optics model are limited by the finite accuracy of the BPMs and long term machine reproducibility.

\section{OPTICS UPGRADES}

A few recently performed optics upgrades were motivated by improving both beam quality and machine diagnostics:

\subsection{Bunch Compression at Injection}

Longitudinal bunching in the injector was originally configured in the $5 \mathrm{MeV}$ region. With the polarized source coming into operation we experienced difficulties transporting the beam, because of small tails in the longitudinal beam profile. Particles in these tails were not on the crest of the accelerating wave and were ultimately lost preventing machine operation at high beam current. To alleviate this problem we redesigned the optics in the injector chicane $(56 \mathrm{MeV})$ to create additional bunch compression. A significant advantage of high energy bunching is that the bunching is not affected by the beam space charge. To facilitate that, a new non-isochronous optics with a negative momentum compaction factor of about $-30 \mathrm{~cm}$ was designed and installed in the injection chicane. To perform the bunch compression one needs to shift the RF phase of the main injector linac by about $6^{\circ}$. To avoid problems with focusing changes at the beginning of the linac only the second of two injector cryomodules is shifted in phase. The new configuration significantly improves machine reliability for high current operation.

\subsection{Medium Dispersion Optics in Lower Arcs}

Responding to current instrumentation needs (installation of synchrotron light monitors to resolve the beam energy spread with high resolution) the optics of arcs 1 and 2 was redesigned. The goal was to increase (by a factor of three) the horizontal dispersion and to decrease the horizontal beta function at the middle of both arcs, where the new monitors were supposed to be installed. The new arc optics, with a mirror-symmetric horizontal dispersion pattern, was designed so that it greatly enhances resolution of the beam energy spread and it still does not limit our energy aperture. To preserve tunability of the new optics one needs to allow for independent correction of both the horizontal dispersion and $\mathrm{M}_{56}$. This was accomplished by appropriate tailoring of the horizontal betatron phase advance inside the arc, to provide two pairs of orthogonal knobs (quadrupoles): for dispersion and momentum compaction adjustments. Furthermore, a betatron wave excited by the first tuning quad, which propagates with twice the betatron frequency, is cancelled by the second wave launched by the remaining quad in the pair, so the net betatron wave is confined to the tuning region and subsequently the tuning process does not affect the betatron match outside the arc.

\section{CONCLUSIONS}

The presented off-line analysis of routinely measured data has uncovered a number of malfunctioning hardware pieces, e.g. improperly functioning BPMs and quadrupoles. Our analysis resulted in a significantly improved optics model of beam transport for the CEBAF accelerator. The new optics preserves the desired lattice architecture and tunability (betatron match, dispersion and momentum compaction adjustment orthogonality).

Furthermore, motivated by improving beam transport quality and machine diagnostics two major optics upgrades were made. They can be summarized as longitudinal bunch compression at injection and installation of medium dispersion optics in lower arcs.

Finally, the presented approach was proven very successful in scaling optics with energy (frequent energy changes are the standard part of our present operation) and in minimizing required machine tuning.

\section{ACKNOWLEDGEMENTS}

We would like to thank $M$. Tiefenback for fruitful discussions, which resulted in better understanding of magnet reproducibility. Useful discussions and valuable input from all members of the Beam Transport Team are gratefully acknowledged.

\section{REFERENCES}

[1] D. Douglas, et al., Proceedings of the 1989 Particle Accelerator Conference, 557-559

[2] B. Bowling, et al., Proceedings of the 1991 Particle Accelerator Conference, 446-448

[3] V. Lebedev, et al., Nuclear Instruments and Methods in Physics Research A, 408 (1998) 373-379

[4] M. Tiefenback, private communication.

[5] Y. Chao, this proceedings. 